\title{
Multiscale finite element methods for porous media flows and their applications
}

\author{
Y. Efendiev* $\quad$ T. Hou ${ }^{\dagger}$
}

February 11, 2006

\begin{abstract}
In this paper, we discuss some applications of multiscale finite element methods to two-phase immiscible flow simulations in heterogeneous porous media. We discuss some extensions of multiscale finite element methods which take into account some limited global information. These methods are well suited for channelized porous media, where the long-range effects are important. This is typical for some recent benchmark tests, such as the SPE comparative solution project [8], where porous media has a channelized structure. The applications of multiscale finite element methods to inverse problems arisen in subsurface characterization are also discussed in the paper.
\end{abstract}

\section{Introduction}

Subsurface flows, as occur in the production of hydrocarbons as well as in environmental remediation projects, are affected by heterogeneities in a wide range of length scales. It is, therefore, very difficult to resolve numerically all of the scales that impact transport through such systems. Typically, upscaled or multiscale models are employed for such systems. The main idea of upscaling techniques is to form coarse-scale equations with a prescribed analytical form that may differ from the underlying fine-scale equations. In multiscale methods, the fine-scale information is carried throughout the simulation and the coarse-scale equations are generally not expressed analytically, but rather formed and solved numerically.

In this paper, we discuss multiscale finite element method (MsFEM) and its applications to the computations of two-phase flows. MsFEM is first introduced in [22]. The main idea of MsFEM is to incorporate the small-scale information into finite element basis functions and capture their effect on the large scale via finite element computations. Recently, a number of multiscale numerical methods, such as residual free bubbles [5], variational multiscale method [24], multiscale finite element method (MsFEM) [22], two-scale finite element methods [26], two-scale conservative subgrid approaches [2], and heterogeneous multiscale method (HMM) [14] have been proposed. We remark that special basis functions in finite element methods have been used earlier in [4]. The generalized finite element method has also been introduced in [3] using special basis function. Multiscale finite element methodology has been modified and successfully applied to two-phase flow simulations in [25] and later in [7, 1]. Arbogast ([2]) used variational multiscale strategy and constructed a multiscale method for two-phase flow simulations.

On the fine (fully resolved) scale, the subsurface flow and transport of $N$ components can be described in terms of an elliptic (for incompressible systems) pressure equation coupled to a sequence of $N-1$ hyperbolic (in the absence of dispersive and capillary pressure effects) conservation laws. Multiscale finite element type methods are used for upscaling/solving of the pressure equation on a coarse grid. As for the transport equation, we consider two approaches. In the first approach, the transport equation is solved on the coarse grid without any subgrid corrections. In the second approach, the transport equation is solved on the fine grid using reconstructed velocity field. In both cases, the pressure equation is solved using MsFEM and

\footnotetext{
*Department of Mathematics, Texas A\&M University, College Station, TX 77843-3368

${ }^{\dagger}$ Applied Mathematics, Caltech, Pasadena, CA 91125
} 
the basis functions are not updated throughout simulations. First procedure, though, provides very fast simulation technique, it is not accurate compared to the second procedure. However, the first procedure can be efficiently used in inverse problems.

For permeability fields with strong non-local effects, some type of global information can be build into the basis functions. In this paper, we discuss the use of global information in multiscale methods. The main idea of the method is to use the global fine-scale solution at initial time to determine the boundary conditions of the basis functions. This method provides a significant improvement in two-phase flow simulations in porous media where the long-range effects are important. This is typical for some recent benchmark tests, such as the SPE comparative solution project [8], where porous media have a channelized structure. The use of global information allows us to capture the long-range effects more accurately compared to the multiscale finite element methods that use only local information to construct the basis functions. We discuss some analysis of the proposed method to illustrate that the method can indeed capture the long-range effect in channelized media. We would like to note that the global solutions in upscaling procedures have been previously used in [6], which motivated our work. Global information within mixed multiscale finite element methods was first used in [1].

With the increasing interest in accurate prediction of subsurface properties, subsurface characterization based on dynamic data, such as production data, takes on greater importance. Thus, to predict future reservoir performance, the reservoir properties, such as porosity and permeability, need to be conditioned to dynamic data, such as production data. In previous findings several methods have been used for predicting permeability field conditioned to production data. Thus, the problem reduces to the sampling from a complicated multi-modal distribution. This problem is challenging because the permeability field is a function defined on a large number of grid blocks. Markov chain Monte Carlo (MCMC) method and its modifications have been used previously to sample the posterior distribution. The direct MCMC simulations are generally very CPU demanding because each proposal requires solving a forward coupled non-linear partial differential equations over a large time interval. The forward fine-scale problem is usually formulated on a large number of grid blocks, which makes it prohibitively expensive to perform sufficient number of MCMC simulations. In this paper, we discuss how to use inexpensive coarse-scale computations to increase the acceptance rate of MCMC calculations. Here the acceptance rate refers to the ratio between the number of accepted permeability samples and the times of solving the fine-scale non-linear PDE system. In particular, we employ multiscale methods for pressure equations in conjunction with solving the transport equation on a coarse grid (without any subgrid treatment) as an approximate solver to guide proposals and reduce the computational cost. Finally, we would like to note that the main goal of this paper is to tie some of our previous results in a unifying way. For this purpose, we perform some additional computations and explain the links between different modifications and applications of multiscale finite element methods.

The paper is organized as follows. In the next section, we discuss the model problem. In Section 3, we discuss the applications of multiscale finite element methods to two-phase flow simulations. In Section 4, we discuss how to use of simplified upscaled models based on multiscale finite element methods in inverse problems.

\section{Model problem}

We consider two-phase flow in a reservoir $\Omega$ under the assumption that the displacement is dominated by viscous effects; i.e., we neglect the effects of gravity, compressibility, and capillary pressure. Porosity will be considered to be constant. The two phases will be referred to as water and oil, designated by subscripts $w$ and $o$, respectively. We write Darcy's law for each phase as follows:

$$
v_{j}=-\frac{k_{r j}(S)}{\mu_{j}} k \nabla p
$$

where $v_{j}$ is the phase velocity, $k$ is the permeability tensor, $k_{r j}$ is the relative permeability to phase $j$ $(j=o, w), S$ is the water saturation (volume fraction), $p$ is pressure and $\mu_{j}$ is the viscosity of phase $j$ $(j=o, w)$. In this work, a single set of relative permeability curves is used and $k$ is assumed to be a diagonal 
tensor. Combining Darcy's law with a statement of conservation of mass allows us to express the governing equations in terms of the so-called pressure and saturation equations:

$$
\begin{gathered}
\nabla \cdot(\lambda(S) k \nabla p)=q, \\
\frac{\partial S}{\partial t}+v \cdot \nabla f(S)=0,
\end{gathered}
$$

where $\lambda$ is the total mobility, $f$ is the fractional flow of water, $q$ is a source term and $v$ is the total velocity, which are given by:

$$
\begin{gathered}
\lambda(S)=\frac{k_{r w}(S)}{\mu_{w}}+\frac{k_{r o}(S)}{\mu_{o}}, \quad f(S)=\frac{k_{r w}(S) / \mu_{w}}{k_{r w}(S) / \mu_{w}+k_{r o}(S) / \mu_{o}}, \\
v=v_{w}+v_{o}=-\lambda(S) k \cdot \nabla p .
\end{gathered}
$$

The above descriptions are referred to as the fine model of the two-phase flow problem. Typical boundary conditions for (2.2) considered in this paper are fixed pressure at some portions of the boundary and no-flow on the rest of the boundary. For the saturation equation (2.3), we impose $S=1$ on the inflow boundaries.

\section{Multiscale methods}

\subsection{Multiscale finite element methods for two-phase flow simulations}

In this section, we will discuss the applications of multiscale finite element methods to two-phase flow simulations. We will use the multiscale finite element framework, though a finite volume element method is chosen as a global solver. Finite volume method is chosen because, by its construction, it satisfies the numerical local conservation which is important in groundwater and reservoir simulations. Let $\mathcal{K}^{h}$ denote the collection of coarse elements/rectangles $K$. Consider a coarse element $K$, and let $\xi_{K}$ be its center. The element $K$ is divided into four rectangles of equal area by connecting $\xi_{K}$ to the midpoints of the element's edges. We denote these quadrilaterals by $K_{\xi}$, where $\xi \in Z_{h}(K)$, are the vertices of $K$. Also, we denote $Z_{h}=\bigcup_{K} Z_{h}(K)$ and $Z_{h}^{0} \subset Z_{h}$ the vertices which do not lie on the Dirichlet boundary of $\Omega$. The control volume $V_{\xi}$ is defined as the union of the quadrilaterals $K_{\xi}$ sharing the vertex $\xi$.

The key idea of the method is the construction of basis functions on the coarse grids, such that these basis functions capture the small-scale information on each of these coarse grids. The method that we use follows its finite element counterpart presented in [22]. The basis functions are constructed from the solution of the leading order homogeneous elliptic equation on each coarse element with some specified boundary conditions. We consider a coarse element $K$ that has $d$ vertices, the local basis functions $\phi_{i}, i=1, \cdots, d$ are set to satisfy the following elliptic problem:

$$
\begin{aligned}
-\nabla \cdot\left(k \cdot \nabla \phi_{i}\right) & =0 \quad \text { in } K \\
\phi_{i} & =g_{i} \quad \text { on } \partial K,
\end{aligned}
$$

for some function $g_{i}$ defined on the boundary of the coarse element $K$. Hou et al. [22] have demonstrated that a careful choice of boundary conditions would improve the accuracy of the method. In previous findings, the function $g_{i}$ for each $i$ is chosen to vary linearly along $\partial K$ or to be the solution of the local one-dimensional problems [25] or the solution of the problem in a slightly larger domain is chosen to define the boundary conditions. In this paper, we will consider linear boundary conditions and also discuss the boundary conditions obtained from a global solution. We will require $\phi_{i}\left(x_{j}\right)=\delta_{i j}$. Finally, a nodal basis function associated with the vertex $x_{i}$ in the domain $\Omega$ is constructed from the combination of the local basis functions that share this $x_{i}$ and zero elsewhere. We would like to note that one can use an approximate solution of (3.1) when it is possible. For example, in the case of periodic or random homogeneous cases, the basis functions can be approximated using homogenization expansion $\phi_{i}=\phi_{i}^{0}+\epsilon N_{k} \nabla_{k} \phi_{i}^{0}$, where $N_{k}$ is the solution of the cell problem and $\phi_{i}^{0}$ is standard finite element basis on the coarse mesh (see [17]). This type of simplification is not applicable for problems considered in this paper. 
Next, we denote by $V^{h}$ the space of our approximate pressure solution, which is spanned by the basis functions $\left\{\phi_{j}\right\}_{x_{j} \in Z_{h}^{0}}$. Then we formulate the finite dimensional problem corresponding to finite volume element formulation of (2.2). A statement of mass conservation on a coarse-control volume $V_{x}$ is formed from (2.2), where the approximate solution is written as a linear combination of the basis functions. Assembly of this conservation statement for all control volumes would give the corresponding linear system of equations that can be solved accordingly. The resulting linear system has incorporated the fine-scale information through the involvement of the nodal basis functions on the approximate solution. To be specific, the problem now is to seek $p^{h} \in V^{h}$ with $p^{h}=\sum_{x_{j} \in Z_{h}^{0}} p_{j} \phi_{j}$ such that

$$
\int_{\partial V_{\xi}} \lambda(S) k \cdot \nabla p^{h} \cdot n d l=0
$$

for every control volume $V_{\xi} \subset \Omega$. Here $n$ defines the normal vector on the boundary of the control volume, $\partial V_{\xi}$ and $S$ is the fine-scale saturation field at this point. The resulting multiscale method differs from the multiscale finite element method, since it employs the finite volume element method as a global solver, and it is called multiscale finite volume element method (MsFVEM). We would like to note that the coarse-scale velocity field obtained using MsFVEM is conservative in control volume elements $V_{\xi}$ (not in $\mathcal{K}^{h}$ ). Due to Dirichlet boundary conditions, the fine-scale velocity field is not conservative, in general. We employ local solvers with Neumann boundary conditions to obtain conservative fine-scale velocity field everywhere. In general, one can resort to simpler reconstruction steps.

In this paper, we will consider two procedures for solving the saturation equation. One of them is a simple/primitive model, where only the coarse scale velocity is used to update the saturation field on the coarse grid, i.e.,

$$
\frac{\partial \bar{S}}{\partial t}+\bar{v} \cdot \nabla f(\bar{S})=0
$$

Here $\bar{v}$ denotes the coarse-scale velocity field obtained using MsFVEM. In (3.3), no upscaling of the saturation equation is performed. These kinds of upscaling techniques in conjunction with the upscaling of absolute permeability is commonly used in applications (see e.g. $[13,12,11]$ ). The difference of our approach is that the coupling of the small scales is performed through the finite volume element formulation of the global problem and the small scale information of the velocity field can be easily recovered. The recovery of small scale information is important if one uses the upscaled models for the saturation equation involving macro-diffusion term. The macro-diffusion term contains two-point correlation function and its calculation requires the fine-scale velocity. We note that the saturation equation is solved on dual grid, because the coarse-scale velocity field is conservative on dual grid. If the saturation profile is smooth this approximation is of first order. In the coarse blocks where the discontinuities of $S$ are present we need to modify the stiffness matrix corresponding to these blocks. The latter requires the values of the fine scale saturation. In a simple upscaling framework, we can not do this because the fine-scale features of the saturation are not available. Instead, we simply use $\lambda(\bar{S})$ in (3.2). This approximation introduces errors. We would like to note that this simple procedure is inexpensive.

In the second approach, the saturation equation is solved on the fine-grid using the reconstructed finescale velocity field. These types of approaches have been used previously, e.g., [23] (cf. [25]). When using MsFVEM for two-phase flow, one can update the basis functions near the sharp fronts. Indeed, sharp fronts modify the local heterogeneities and this can be taken into account by re-solving the local equations, (3.1), for basis functions. If the saturation is smooth in the coarse block, it can be approximated by its average in (3.1), and consequently, the basis functions are not needed to be updated. It can be shown that this approximation yields first-order errors (in terms of coarse mesh size). In our simulations, we have found only a slight improvement if the basis functions are updated, thus the numerical results for MsFVEM presented in this paper do not include the basis function update near the sharp fronts. We would like to note that the basis function update becomes more important for the cases $\mu_{0} / \mu_{w}<<1$ (i.e., water phase viscosity is much smaller than oil phase viscosity). In this paper, we consider the cases $\mu_{0} / \mu_{w}>1$. For two-phase flow simulations, we will use IMPES formulation (implicit pressure and explicit saturation) for the computations. At each time the velocity is computed. Then the velocity is used to update the saturation. 


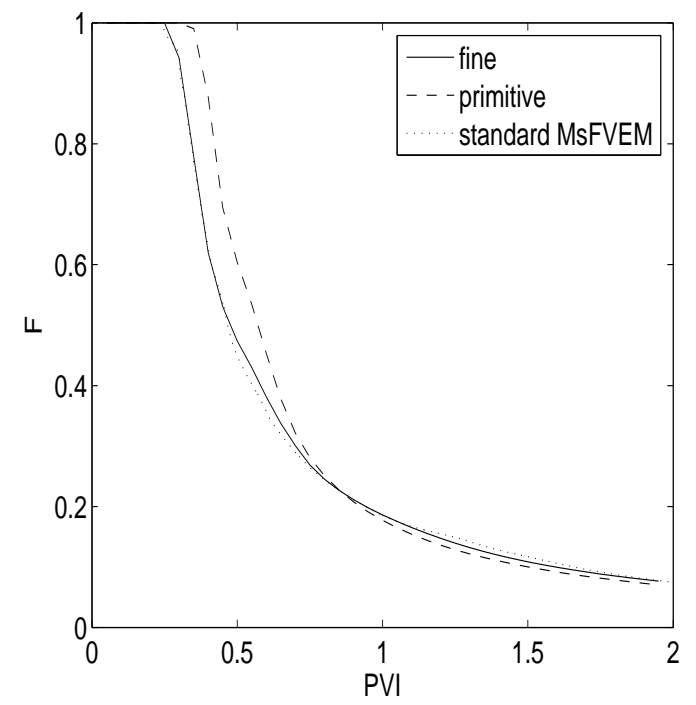

Figure 1: Fractional flow comparison for a permeability field generated using two-point geostatistics.

We note that the upscaling of transport equation, which is purely hyperbolic, is difficult, in general. There are a number of upscaling methodologies are developed for the transport equation arisen in porous media flows, e.g., [30, 31, 16, 15]. However, we will not be using any subgrid treatment for the transport equation in this paper.

Next, we present a representative numerical example for a permeability field generated using two-point geostatistics. To generate this permeability field, we have used GSLIB algorithm [9]. The permeability is $\log$-normally distributed with prescribed variance $\sigma^{2}=1.5\left(\sigma^{2}\right.$ here refers to the variance of $\left.\log k\right)$ and some correlation structure. The correlation structure is specified in terms of dimensionless correlation lengths in the $x$ and $z$-directions, $l_{x}=0.4$ and $l_{z}=0.04$, nondimensionalized by the system length. Linear boundary conditions are used for constructing multiscale basis function in (3.1). Spherical variogram is used [9]. In this numerical example, the fine-scale field is $120 \times 120$, while the coarse-scale field is $12 \times 12$ defined in the rectangle with the length 5 and the width 1 . For the two-phase flow simulations, the system is considered to initially contain only oil $(S=0)$ and water is injected at inflow boundaries $(S=1$ is prescribed), i.e., we specify $p=1, S=1$ along the $x=0$ edge and $p=0$ along the $x=5$ edge, and no flow boundary conditions on the lateral boundaries. Relative permeability functions are specified as $k_{r w}=S^{2}, k_{r o}=(1-S)^{2}$; water and oil viscosities are set to $\mu_{w}=1$ and $\mu_{o}=5$. Porosity is constant and serves only to nondimensionalize time. Results are presented in terms of the fraction of oil in the produced fluid (i.e., oil cut, designated $F$ ) against pore volume injected (PVI). PVI represents dimensionless time and is computed via $\int Q d t / V_{p}$ where $V_{p}$ is the total pore volume of the system and $Q$ is the total flow rate.

In our first numerical test, Figure 1, we compare the fractional flows. The dashed line corresponds to the calculations performed using a simple saturation upscaling (no subgrid treatment), while dotted line corresponds to the calculations performed by solving the saturation equation on the fine grid using the reconstructed fine-scale velocity field. We observe from this figure that the second approach is very accurate, while the first approach over-predicts the breakthrough time. We have also observed very good agreement between the saturation fields when the second approach for solving the saturation equation is used.

In the next set of numerical results, we consider strongly channelized permeability fields. These permeability fields are proposed in some recent benchmark tests, such as the SPE comparative solution project [8]. In Figure 2, one of the layers of this 3-D permeability field is depicted. All the layers have $220 \times 60$ fine-scale resolution, and we take the coarse grid to be $22 \times 6$. As it can be observed, the permeability field contains a high permeability channel, where most flow will occur in our simulation. In Figure 3, the fractional flows 


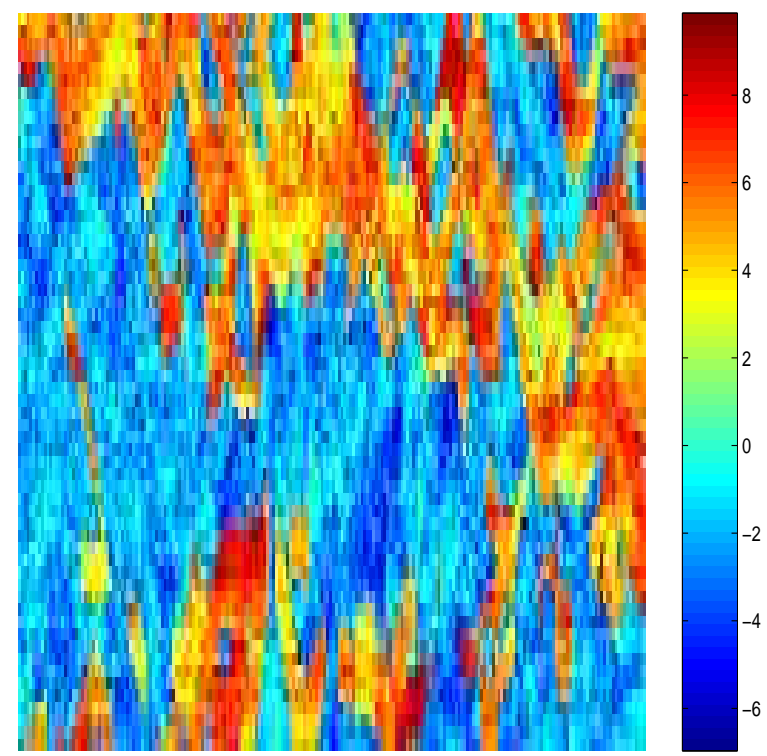

Figure 2: Log-permeability for one of the layers of upper Ness.

are compared. The boundary conditions are taken to be $p=1, S=1$ along the $x=0$ edge and $p=0$ along the $x=5$ edge, and no flow boundary conditions on the lateral boundaries. Again, the dashed line corresponds to the calculations performed using a simple saturation upscaling (no subgrid treatment), while dotted line corresponds to the calculations performed by solving the saturation equation on the fine grid using the reconstructed fine-scale velocity field. We observe from this figure that the second approach is not very accurate in contrast to the permeability field generated using two-point geostatistics [9]. This is because the local basis functions can not account accurately the global connectivity of the media. Indeed, in the next figure, Figure 4, the saturation fields at time PVI $=0.5$ are compared. We see that multiscale finite element methods with local basis functions introduce some errors. In the bottom left corner, there is a saturation pocket which is not in the reference solution computed using a fine grid. The reason for this is that the local basis functions in the lower left corner contains high permeability region. However, this high permeability region does not have global connectivity, and the local basis functions can not take this effect into account. Next, we discuss how global information can be incorporated into multiscale basis functions to improve the accuracy of the computations.

\subsection{Multiscale methods using limited global information}

The main idea of the modified multiscale finite volume element method (MsFVEM) is to use the solution of the fine-scale problem at time zero to determine the boundary conditions for the basis functions. This approach is proposed in [18] to handle the permeability fields which are strongly channelized. For this type of permeability fields, some type of global information is needed. Next, we describe the method. We denote the solution of $(2.2)$ at time zero by $p^{\text {init }}(x)$. In defining $p^{i n i t}(x)$, we use the actual boundary conditions of the global problem. $p^{\text {init }}(x)$ depends on global boundary conditions, and, generally, is updated each time when global boundary conditions are changed. The boundary conditions in (3.1) for modified basis functions are defined in the following way. For each rectangular element $K$ with vertices $x_{i}(i=1,2,3,4)$ denote by $\phi_{i}(x)$ a restriction of the nodal basis on $K$, such that $\phi_{i}\left(x_{j}\right)=\delta_{i j}$. At the edges where $\phi_{i}(x)=0$ at both vertices, we take boundary condition for $\phi_{i}(x)$ to be zero. Consequently, the basis functions are localized. We only need to determine the boundary condition at two edges which have the common vertex $x_{i}\left(\phi_{i}\left(x_{i}\right)=1\right)$. Denote these two edges by $\left[x_{i-1}, x_{i}\right]$ and $\left[x_{i}, x_{i+1}\right]$ (see Figure 5 ). We only need to describe the boundary condition, 


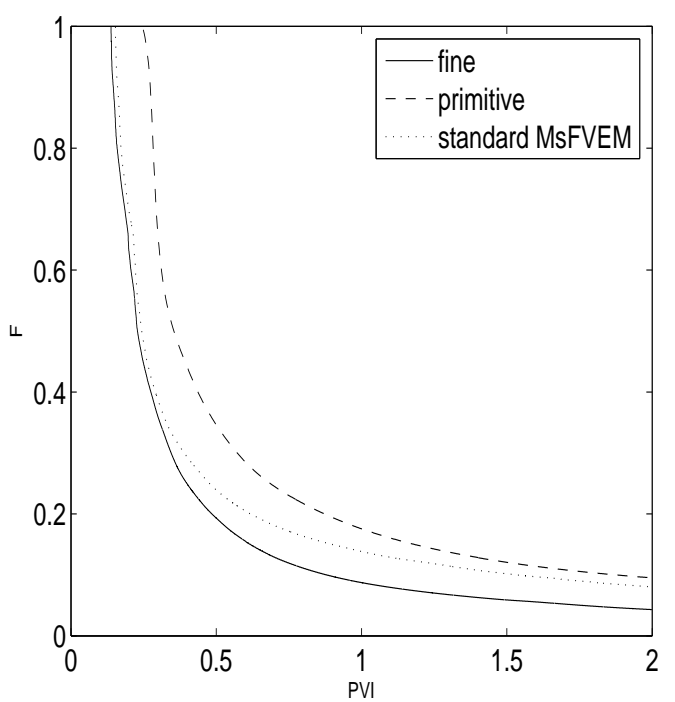

Figure 3: Fractional flow comparison for a channelized permeability field.
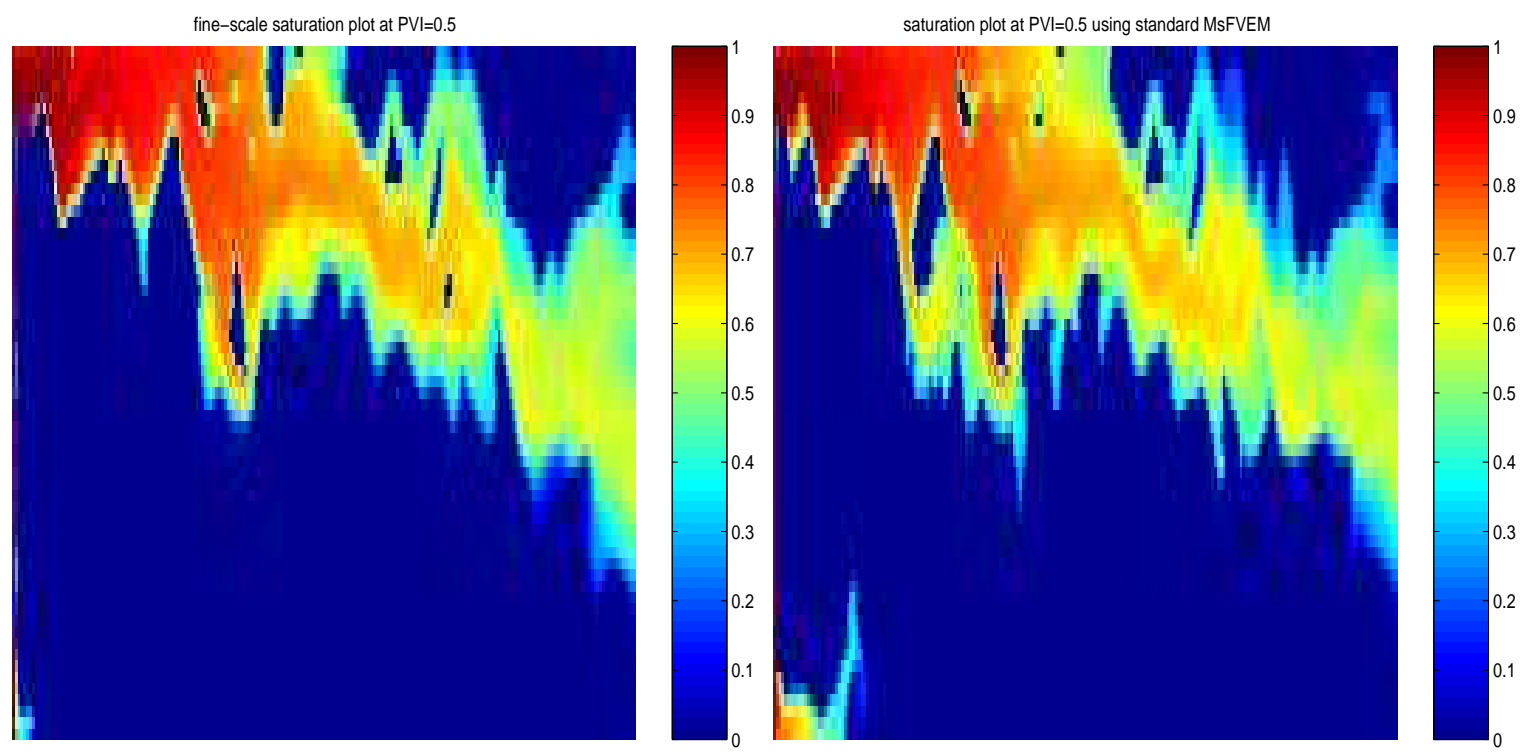

Figure 4: Saturation maps at PVI=0.5 for fine-scale solution (left figure) and standard MsFVEM (right figure). 


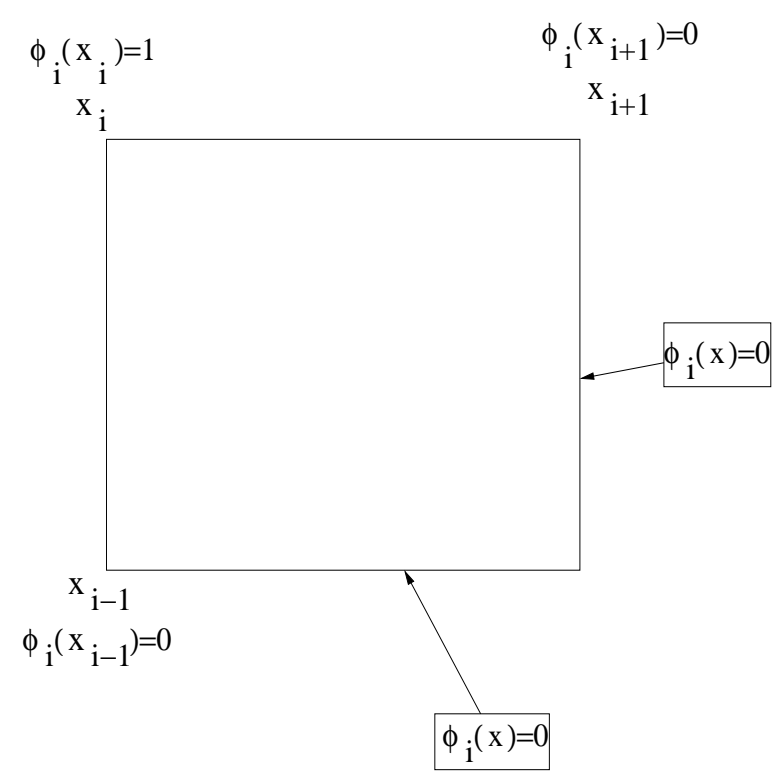

Figure 5: Schematic description of nodal points.

$g_{i}(x)$, for the basis function $\phi_{i}(x)$, along the edges $\left[x_{i}, x_{i+1}\right]$ and $\left[x_{i}, x_{i-1}\right]$. If $p^{\text {init }}\left(x_{i}\right) \neq p^{\text {init }}\left(x_{i+1}\right)$, then

$$
\left.g_{i}(x)\right|_{\left[x_{i}, x_{i+1}\right]}=\frac{p^{i n i t}(x)-p^{i n i t}\left(x_{i+1}\right)}{p^{i n i t}\left(x_{i}\right)-p^{i n i t}\left(x_{i+1}\right)},\left.\quad g_{i}(x)\right|_{\left[x_{i}, x_{i-1}\right]}=\frac{p^{\text {init }}(x)-p^{i n i t}\left(x_{i-1}\right)}{p^{i n i t}\left(x_{i}\right)-p^{i n i t}\left(x_{i-1}\right)} .
$$

If $p^{\text {init }}\left(x_{i}\right)=p^{\text {init }}\left(x_{i+1}\right) \neq 0$, then

$$
\left.g_{i}(x)\right|_{\left[x_{i}, x_{i+1}\right]}=\phi_{i}^{0}(x)+\frac{1}{2 p^{i n i t}\left(x_{i}\right)}\left(p^{i n i t}(x)-p^{i n i t}\left(x_{i+1}\right)\right),
$$

where $\phi_{i}^{0}(x)$ is a linear function on $\left[x_{i}, x_{i+1}\right]$ such that $\phi_{i}^{0}\left(x_{i}\right)=1$ and $\phi_{i}^{0}\left(x_{i+1}\right)=0$. Similarly,

$$
\left.g_{i+1}(x)\right|_{\left[x_{i}, x_{i+1}\right]}=\phi_{i+1}^{0}(x)+\frac{1}{2 p^{i n i t}\left(x_{i+1}\right)}\left(p^{i n i t}(x)-p^{i n i t}\left(x_{i+1}\right)\right),
$$

where $\phi_{i+1}^{0}(x)$ is a linear function on $\left[x_{i}, x_{i+1}\right]$ such that $\phi_{i+1}^{0}\left(x_{i+1}\right)=1$ and $\phi_{i+1}^{0}\left(x_{i}\right)=0$. If $p^{\text {init }}\left(x_{i}\right)=$ $p^{i n i t}\left(x_{i+1}\right) \neq 0$, then one can also use simply linear boundary conditions. If $p^{\text {init }}\left(x_{i}\right)=p^{\text {init }}\left(x_{i+1}\right)=0$ then linear boundary conditions are used. In the applications considered in this paper, the initial pressure is always positive. Finally, the basis function $\phi_{i}(x)$ is constructed by solving (3.1). The choice of the boundary conditions for the basis functions is motivated by the analysis. In particular, we would like to recover the exact fine-scale solution along each edge if the nodal values of the pressure are equal to the values of exact fine-scale pressure. This is the underlying idea for the choice of boundary conditions. Using this property and Cea's lemma one can show that the pressure obtained from the numerical solution is equal to the underlying fine-scale pressure.

We have proposed some analysis for modified multiscale finite element method in [18]. The main idea is to show that the pressure evolution in two-phase flow simulations is strongly influenced by the initial pressure. To demonstrate this, we consider a channelized permeability field, where the value of the permeability in the channel is large. We assume the permeability has the form $k I$, where $I$ is an identity matrix. In a channelized medium, the dominant flow is within the channels. Our analysis assumes a single channel and restricted to 2-D. Here, we briefly mention the main findings. Denote the initial stream function and pressure by $\eta=\psi(x, t=0)$ and $\zeta=p(x, t=0)$ ( $\zeta$ is also denoted by $p^{i n i t}$ previously). The stream function is defined

$$
\partial \psi / \partial x_{1}=-v_{2}, \quad \partial \psi / \partial x_{2}=v_{1} .
$$


Then the equation for the pressure can be written as

$$
\frac{\partial}{\partial \eta}\left(|k|^{2} \lambda(S) \frac{\partial p}{\partial \eta}\right)+\frac{\partial}{\partial \zeta}\left(\lambda(S) \frac{\partial p}{\partial \zeta}\right)=0 .
$$

For simplicity, $S=0$ at time zero is assumed. We consider a typical boundary condition that gives high flow within the channel, such that the high flow channel will be mapped into a large slab in $(\eta, \zeta)$ coordinate system. If the heterogeneities within the channel in $\eta$ direction is not strong (e.g., narrow channel in Cartesian coordinates), the saturation within the channel will depend on $\zeta$. In this case, the leading order pressure will depend only on $\zeta$, and it can be shown that

$$
p(\eta, \zeta, t)=p_{0}(\zeta, t)+\text { high order terms },
$$

where $p_{0}(\zeta, t)$ is the dominant pressure. This asymptotic expansion shows that the time-varying pressure strongly depends on the initial pressure (i.e., the leading order term in the asymptotic expansion is a function of initial pressure and time only). Because the global basis functions can recover the initial pressure exactly, the modified basis can capture the global pressure more accurately. We would like to note that one can also show that the dominant velocity field is $v_{\zeta}=\lambda(S) \frac{\partial p}{\partial \zeta}$.

Next, we show the numerical results obtained using modified multiscale finite element type methods for the permeability layer depicted in Figure 2 and two-phase flow parameters presented earlier. We consider two types of boundary conditions in a rectangular region $[0,5] \times[0,1]$. For the first type of boundary conditions, we specify $p=1, S=1$ along the $x=0$ edge and $p=0$ along the $x=5$ edge. On the rest of the boundaries, we assume no flow boundary condition. We call this type of the boundary condition as side-to-side. The other type of boundary conditions is obtained by specifying $p=1, S=1$ along the $x=0$ edge for $0.5 \leq z \leq 1$ and $p=0$ along the $x=5$ edge for $0 \leq z \leq 0.5$. On the rest of the boundaries, we assume no flow boundary condition.

In Figure 6, the fractional flows are plotted for standard and modified MsFVEM. We observe from this figure that modified MsFVEM is more accurate and provides nearly the same fractional flow response as the direct fine-scale calculations. In Figure 7, we compare the saturation fields at PVI=0.5. As we see, the saturation field obtained using modified MsFVEM is very accurate and there is no longer the saturation pocket at the left bottom corner. Thus, the modified MsFVEM captures the connectivity of the media accurately.

In the next set of numerical results, we test the modified multiscale finite element methods for a different layer (layer 40) of SPE comparative solution project. In Figure 8 and 9, the fractional flows and total flow rates $(Q)$ are compared for two different boundary conditions. One can see clearly that the modified MsFVEM method gives nearly exact results for these integrated responses. The standard MsFVEM tends to over-predict the total flow rate at time zero. This initial error persists at later times. This phenomena is often observed in upscaling of two-phase flows. More numerical results and discussions can be found in [18]. These numerical results demonstrate that modified multiscale finite element methods which use a limited global information are more accurate. Moreover, modified multiscale finite element methods are capable of capturing the long-range flow features accurately for channelized permeability fields.

\section{Applications of multiscale methods to dynamic data integration}

In this section, we present some applications of multiscale finite element methods to inverse problems. The results described in this section summarize the results presented in $[19,10]$. The problem under consideration consists of sampling permeability field given fractional flow measurements. Here, by measurements we mean the fractional flow data given as a function of time. Typically, it is assumed that the permeability field is known at some locations. This information can be incorporated into the prior models (distributions). Since the fractional flow is an integrated response, the map from the permeability field to the fractional flow is not one-to-one. Hence this problem is ill-posed in the sense that there exist many different permeability realizations for the given production data. 


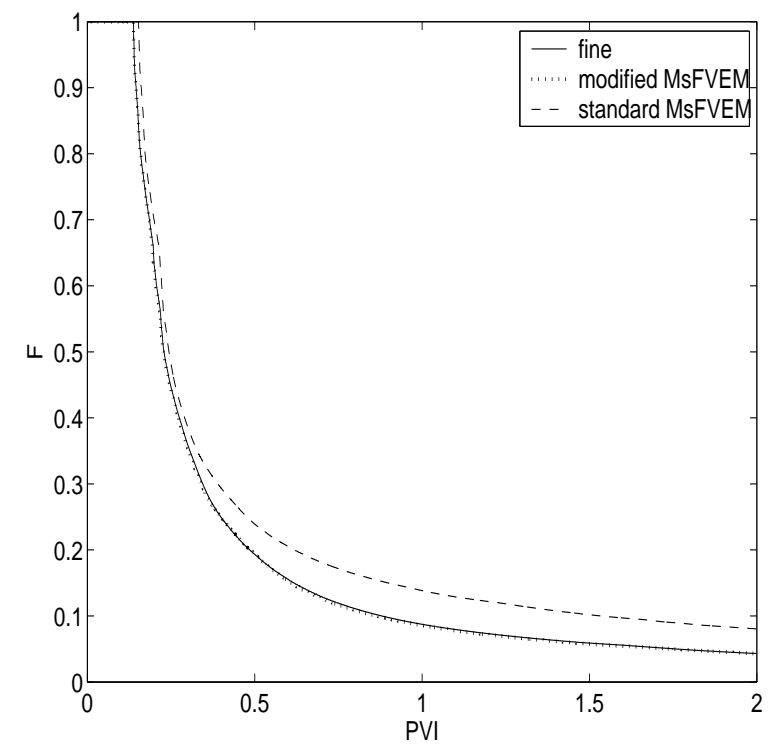

Figure 6: Fractional flow comparison for standard MsFVEM and modified MsFVEM for side-to-side flow.
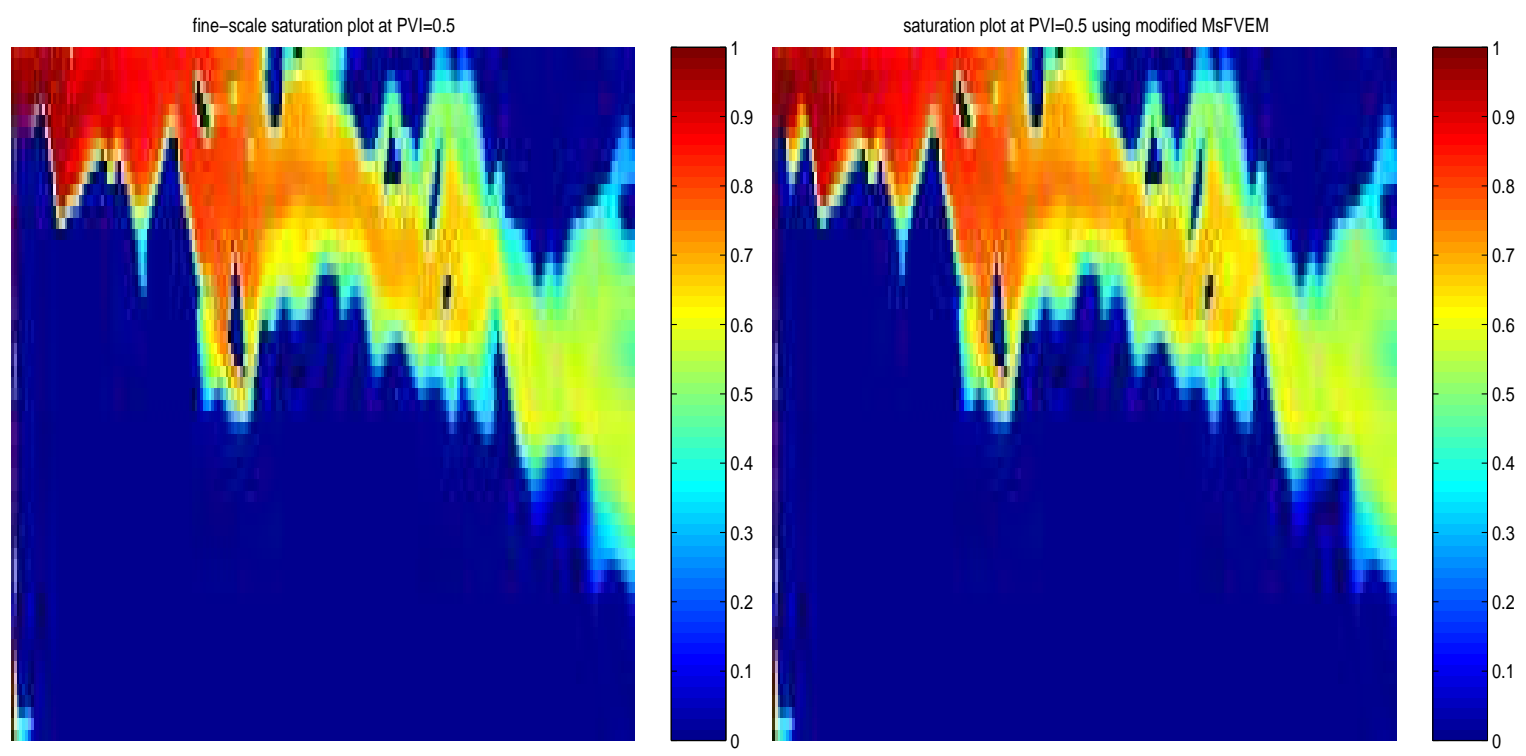

Figure 7: Saturation maps at PVI=0.5 for fine-scale solution (left figure) and modified MsFVEM (right figure). Side-to-side boundary condition is used. 

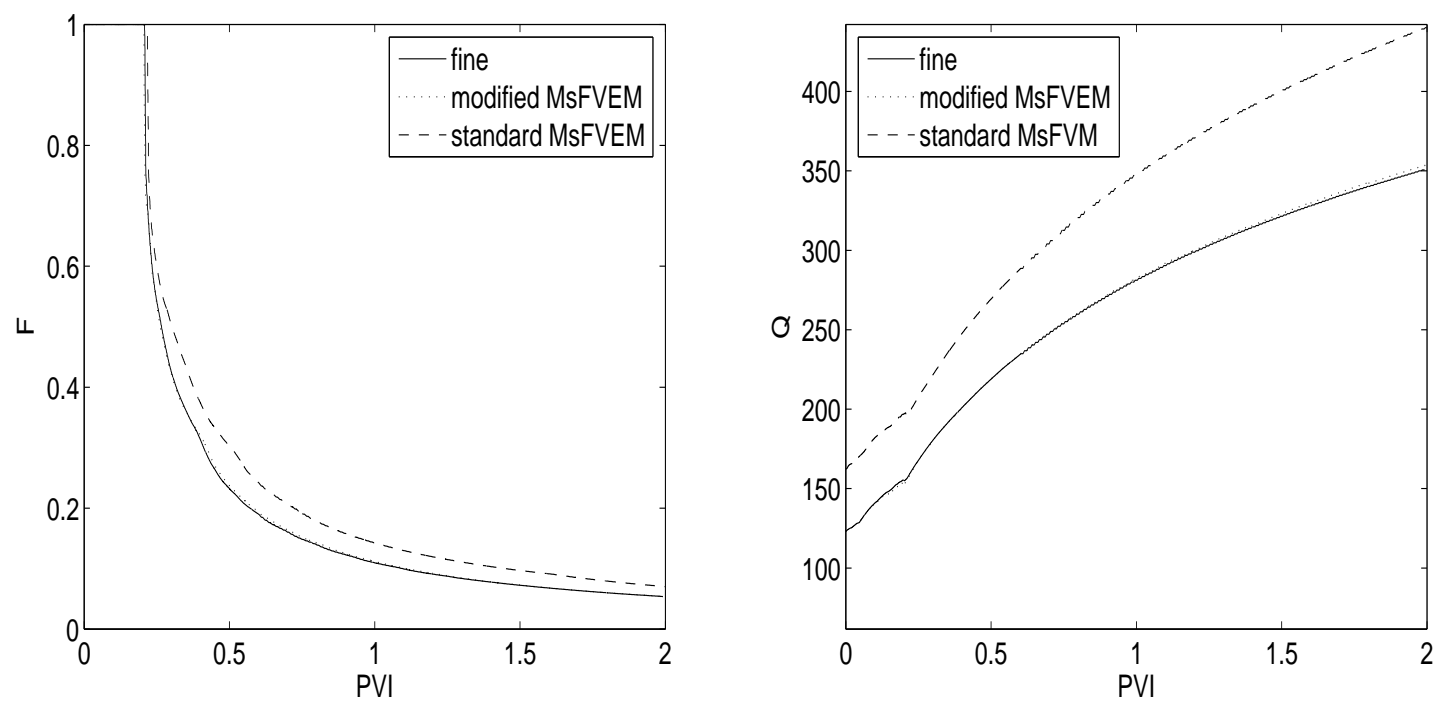

Figure 8: Fractional flow (left figure) and total production (right figure) comparison for standard MsFVEM and modified MsFVEM for side-to-side flow (layer 40).
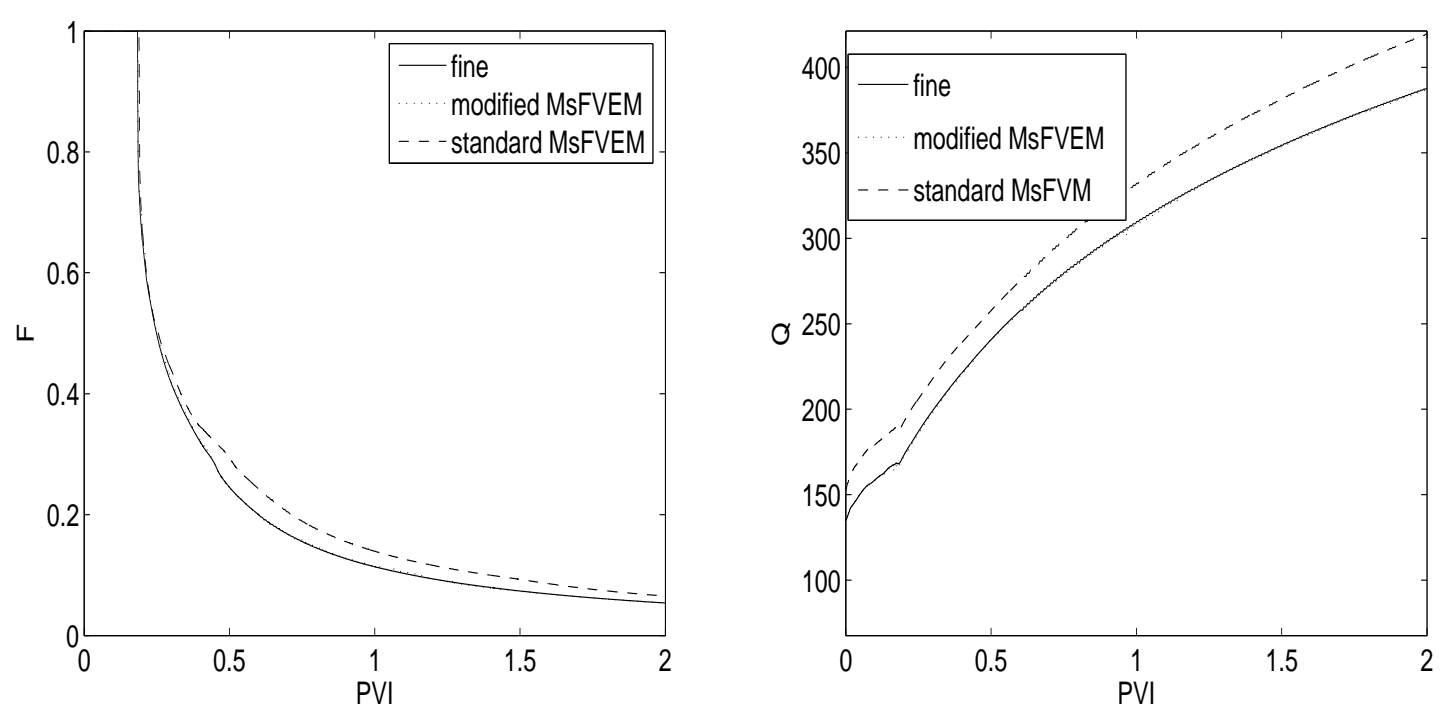

Figure 9: Fractional flow (left figure) and total production (right figure) comparison for standard MsFVEM and modified MsFVEM for corner-to-corner (layer 40). 
From the probabilistic point of view, this problem can be regarded as the conditioning of the permeability field to the fractional flow data with measurement errors. Consequently, our goal is to sample from the conditional distribution $P(k \mid F)$, where $k$ is the fine-scale permeability field and $F$ is the fractional flow curve measured from production data. Using the Bayes theorem we can write

$$
P(k \mid F) \propto P(F \mid k) P(k) .
$$

In the above formula, $P(k)$ is the unconditioned (prior) distribution of the permeability field, which is assumed to be log-normal. $P(F \mid k)$ denotes the conditional probability that the outcome of the measurement is $F$ when the true permeability is $k$. In practice, the measured fractional flow contains measurement errors. In this paper, we assume that the measurement error satisfies a Gaussian distribution, thus, the likelihood function $P(F \mid k)$ takes the form

$$
P(F \mid k) \propto \exp \left(-\frac{\left\|F-F_{k}\right\|^{2}}{\sigma_{f}^{2}}\right),
$$

where $F_{k}$ is the fractional flow computed by solving the nonlinear PDE system (2.1)-(2.3) on the fine-grid for the given $k$, and $\sigma_{f}$ is the measurement precision. Since both $F$ and $F_{k}$ are functions of $t$, we take the norm $\left\|F-F_{k}\right\|^{2}$ to be the $L_{2}$ norm, i.e.,

$$
\left\|F-F_{k}\right\|^{2}=\int_{0}^{T}\left(F(t)-F_{k}(t)\right)^{2} d t .
$$

We note that the methods discussed in this paper are not limited to Gaussian error functions, and any general covariance describing measurement errors can be used in the simulations. We also note that different permeability fields may produce the same fractional flow curve, the likelihood distribution $P(F \mid k)$ is a multi-modal function of $k$ (i.e. has multiple local maxima).

Denote

$$
\pi(k)=P(k \mid F) \propto \exp \left(-\frac{\left\|F-F_{k}\right\|^{2}}{\sigma_{f}^{2}}\right) P(k) .
$$

Sampling from the distribution $\pi(k)$ can be accomplished by using the Markov chain Monte Carlo (MCMC) method. The main idea of MCMC is to generate a Markov chain with $\pi(k)$ as its stationary distribution. A key step to this approach is to construct the desired transition probability distribution for the Markov chain. In this paper, we use the Metropolis-Hasting algorithm. Suppose $q(y \mid x)$ is a general transitional probability distribution, which is easy to sample and has an explicit form. The Metropolis-Hasting MCMC algorithm (see, e.g., [29]) consists of the following steps.

\section{Algorithm (Metropolis-Hasting MCMC [29])}

- Step 1. At $k_{n}$ generate $k$ from $q\left(k \mid k_{n}\right)$.

- Step 2. Accept $k$ as a sample with probability

$$
p\left(k_{n}, k\right)=\min \left(1, \frac{q\left(k_{n} \mid k\right) \pi(k)}{q\left(k \mid k_{n}\right) \pi\left(k_{n}\right)}\right),
$$

i.e. take $k_{n+1}=k$ with probability $p\left(k_{n}, k\right)$, and $k_{n+1}=k_{n}$ with probability $1-p\left(k_{n}, k\right)$.

Starting with an arbitrary initial permeability sample $k_{0}$, the MCMC algorithm generates a Markov chain $\left\{k_{n}\right\}$. At each iteration, the probability of moving from state $k_{n}$ to a next state $k$ is $q\left(k \mid k_{n}\right) p\left(k_{n}, k\right)$, so the transition kernel for the Markov chain $\left\{k_{n}\right\}$ is

$$
K\left(k_{n}, k\right)=p\left(k_{n}, k\right) q\left(k \mid k_{n}\right)+\left(1-\int p\left(k_{n}, k\right) q\left(k \mid k_{n}\right) d k\right) \delta_{k_{n}}(k) .
$$


Using the explicit formula of the transition kernel, it is not difficult to prove that the target distribution $\pi(k)$ is indeed the stationary distribution of the Markov chain $\left\{k_{n}\right\}$. As a result, we can take $k_{n}$ as samples of the distribution $\pi(k)$ after the chain reaches steady state.

In the Metropolis-Hasting MCMC algorithm, the major computational cost is to compute the value of the target distribution $\pi(k)$, which involves solving the coupled non-linear PDE system (2.1)-(2.3) on the fine-grid. Generally, the MCMC method requires thousands of iterations before it converges to the steady state. To quantify the uncertainty of the permeability field accurately, one also needs to generate a large number of different samples. Thus, the direct (full) MCMC simulations are usually prohibitively expensive. Moreover, the acceptance rate of the direct MCMC method is very low (less than 1\%), due to the large dimensions of the permeability field. As a result, most of the CPU time is spent on rejected samples.

An important way to improve the direct MCMC method is to increase the acceptance rate by modifying the proposal distribution $q\left(k \mid k_{n}\right)$. We discuss algorithms that use approximate and inexpensive coarse-scale simulations to speed-up MCMC calculations. In particular, we consider an approach where the saturation equation is upscaled using a simple volume averaging (3.3) and the pressure equation is upscaled using MsFVEM. Though, we have observed that this type of upscaling can introduce large errors, it can be used in dynamic data integration problems for the following reasons. First, this approach which combines MsFVEM for the pressure equation and (3.3) for the saturation equation, is very inexpensive. Secondly, we have observed that there is a strong correlation between fine and coarse-scale fractional flows. Correlation coefficient computed using 5000 independent permeability realizations is approximately 0.9 (cf. [19]). Denote $F^{*}$ as the coarse-scale reference fractional flow curve corresponding to the observed fine-scale one. Then $\pi^{*}(k)=P\left(k \mid F^{*}\right)$ is a coarse-scale approximation to the target distribution $\pi(k)$. Similarly, we have

$$
\pi^{*}(k)=P\left(k \mid F^{*}\right) \propto P\left(F^{*} \mid k\right) P(k) .
$$

As before, we assume a Gaussian error model for the likelihood function $P\left(F^{*} \mid k\right)$, that is

$$
P\left(F^{*} \mid k\right) \propto \exp \left(-\frac{\left\|F^{*}-F_{k}^{*}\right\|^{2}}{\sigma_{c}^{2}}\right),
$$

where $F_{k}^{*}$ is the fractional flow curve computed by solving the coarse-scale model of (2.1)-(2.3) for the given

$k$, and $\sigma_{c}$ is the precision associated with the reference coarse-scale fractional flow $F^{*} . \sigma_{c}$ is usually taken to be slightly larger than $\sigma_{f}$ (see [19]). Using the coarse-scale distribution $\pi^{*}(k)$ as a filter, the preconditioned MCMC can be described as following ([19]).

\section{Algorithm I (preconditioned MCMC)}

- Step 1. At $k_{n}$, generate a trial proposal $k^{\prime}$ from distribution $q\left(k^{\prime} \mid k_{n}\right)$.

- Step 2. Take the proposal as

$$
k= \begin{cases}k^{\prime} & \text { with probability } g\left(k_{n}, k^{\prime}\right), \\ k_{n} & \text { with probability } 1-g\left(k_{n}, k^{\prime}\right),\end{cases}
$$

where

$$
g\left(k_{n}, k^{\prime}\right)=\min \left(1, \frac{q\left(k_{n} \mid k^{\prime}\right) \pi^{*}\left(k^{\prime}\right)}{q\left(k^{\prime} \mid k_{n}\right) \pi^{*}\left(k_{n}\right)}\right) .
$$

Here $\pi^{*}(k)$ represents the coarse-scale approximation to the target distribution $\pi(k)$. Therefore, the final proposal $k$ is generated from the effective instrumental distribution

$$
Q\left(k \mid k_{n}\right)=g\left(k_{n}, k\right) q\left(k \mid k_{n}\right)+\left(1-\int g\left(k_{n}, k\right) q\left(k \mid k_{n}\right) d k\right) \delta_{k_{n}}(k) .
$$


- Step 3. Accept $k$ as a sample with probability

$$
\rho\left(k_{n}, k\right)=\min \left(1, \frac{Q\left(k_{n} \mid k\right) \pi(k)}{Q\left(k \mid k_{n}\right) \pi\left(k_{n}\right)}\right),
$$

i.e. $k_{n+1}=k$ with probability $\rho\left(k_{n}, k\right)$, and $k_{n+1}=k_{n}$ with probability $1-\rho\left(k_{n}, k\right)$.

There are a number of facts we would like to note about the preconditioned MCMC algorithm. If $k^{\prime}$ is rejected by the coarse-scale test ( $\rho\left(k_{n}, k_{n}\right) \equiv 1$, no further (fine-scale) computation is needed and $k_{n}$ will be accepted for one more time. On the contrary, the regular MCMC method requires a fine-scale simulation for every proposal $k$, even though it is rejected. Furthermore, there is no need to compute $Q\left(k \mid k_{n}\right)$ and $Q\left(k_{n} \mid k\right)$ in (4.8) by formula (4.7). The acceptance probability(4.8) can be simplified as

$$
\rho\left(k_{n}, k\right)=\min \left(1, \frac{\pi(k) \pi^{*}\left(k_{n}\right)}{\pi\left(k_{n}\right) \pi^{*}(k)}\right) .
$$

Since the computation of the coarse-scale solution is very cheap, the step 2 can be implemented very fast to decide whether or not to run fine-scale simulations. The second step of the algorithm serves as a filter that avoids unnecessary fine-scale runs for the rejected samples. In [19], we show that the modified Markov chain is ergodic and converges to the correct distribution.

Next, we demonstrate some numerical examples. Suppose the permeability field $k(x)$ is defined on the unit square $\Omega=[0,5] \times[0,1]$. We assume that the permeability field $k$ is known at some spatial locations and that the covariance of the permeability is also known. We discretize the domain $\Omega$ by a rectangular mesh and hence the permeability field $k$ is represented by a matrix (thus $k$ is a high dimensional vector). As for the boundary conditions, we have tested various boundary conditions and observed similar results for the preconditioned MCMC. In our numerical experiments we will assume side-to-side boundary conditions, $p=1$ and $S=1$ on $x=0$ and $p=0$ on $x=5$, and no flow boundary conditions on the lateral boundaries. We have chosen this type of boundary conditions because they provide a large deviation between coarseand fine-scale simulations for permeability fields considered in the paper.

Using the Karhunen-Loeve expansion [32], the permeability field can be expanded in terms of an optimal $L^{2}$ basis. By truncating the expansion we can represent the permeability matrix by a small number of random parameters. To impose the hard constraints (the values of the permeability at prescribed locations), we will find a linear subspace of our parameter space (a hyperplane) which yields the corresponding values of the permeability field. Denote $Y(x, \omega)=\log [k(x, \omega)]$, where $\omega$ denoting an elementary random event is included to remind us that $k$ is a random field. Suppose $Y(x, \omega)$ is a second order stochastic process, that is, $Y(x, \omega) \in L^{2}(\Omega)$ with probability one. We will assume that $E[Y(x, \omega)]=0$. Denote the covariance function of $Y$ as $R(x, y)=E[Y(x) Y(y)]$. Then, there exist basis functions $\left\{\phi_{k}\right\}$ that satisfy

$$
\int_{\Omega} R(x, y) \phi_{k}(y) d y=\lambda_{k} \phi_{k}(x), \quad k=1,2, \ldots,
$$

where $\lambda_{k}=E\left[Y_{k}^{2}\right]>0$. Furthermore, we have

$$
R(x, y)=\sum_{k=1}^{\infty} \lambda_{k} \phi_{k}(x) \phi_{k}(y)
$$

Denote $\theta_{k}=Y_{k} / \sqrt{\lambda_{k}}$, then $\theta_{k}$ satisfy $E\left(\theta_{k}\right)=0$ and $E\left(\theta_{i} \theta_{j}\right)=\delta_{i j}$. It follows that

$$
Y(x, \omega)=\sum_{k=1}^{\infty} \sqrt{\lambda_{k}} \theta_{k}(\omega) \phi_{k}(x)
$$

where $\phi_{k}$ and $\lambda_{k}$ satisfy (4.10). We assume that eigenvalues $\lambda_{k}$ are ordered so that $\lambda_{1} \geq \lambda_{2} \geq \ldots$. The expansion (4.12) is called Karhunen-Loeve expansion (KLE). 
Suppose the permeability field $k(x, \omega)$ is a log normal homogeneous stochastic process, then $Y(x, \omega)$ is a Gaussian process and $\theta_{k}$ are independent standard Gaussian random variables. We assume that the covariance function of $Y(x, \omega)$ bears the form

$$
R(x, y)=\sigma^{2} \exp \left(-\frac{\left|x_{1}-y_{1}\right|^{2}}{2 L_{x}^{2}}-\frac{\left|x_{2}-y_{2}\right|^{2}}{2 L_{y}^{2}}\right) .
$$

In the above formula, $L_{x}$ and $L_{y}$ are the correlation lengths in each dimension, and $\sigma^{2}=E\left(Y^{2}\right)$ is a constant. We first solve the eigenvalue problem (4.10) numerically and obtain the eigenpairs $\left\{\lambda_{k}, \phi_{k}\right\}$. Since eigenvalues decay fast, the truncated KLE should approximate the stochastic process $Y(x, \omega)$ fairly well in $L^{2}$ sense. Therefore, we can sample $Y(x, \omega)$ from the truncated KLE (4.12) by generating Gaussian random variables $\theta_{k}$.

Next, we present some numerical examples to demonstrate the efficiency of preconditioned MCMC methods. More detailed numerical studies of preconditioned MCMC method can be found in [19]. In the simulation, we first generate a reference permeability field using all eigenvectors and compute the corresponding fractional flows. To propose permeability fields from the prior (unconditioned) distribution, we maintain 20 terms in the KLE. Suppose the permeability field is known at 9 distinct points. This condition is imposed by setting

$$
\sum_{k=1}^{20} \sqrt{\lambda_{k}} \theta_{k} \phi_{k}\left(x_{j}\right)=\alpha_{j},
$$

where $\alpha_{j}(j=1, \ldots, 9)$ are prescribed constants. For simplicity, we set $\alpha_{j}=0$ for all $j=1, \ldots, 9$. In the simulations we propose eleven $\theta_{i}$ and calculate the rest of $\theta_{i}$ by solving the linear system (4.14). In all the simulations, we test 50000 proposals and iterate the Markov chain 50000 times.

We have considered two types of instrumental proposal distributions, independent sampler and random walk sampler. In the case of independent samplers, the proposal distribution $q\left(k \mid k_{n}\right)$ is chosen to be independent of $k_{n}$ and equal to the prior (unconditioned) distribution. In the random walk sampler, the proposal distribution depends on the previous value of the permeability field and is given by

$$
q\left(k \mid k_{n}\right)=k_{n}+\epsilon_{n},
$$

where $\epsilon_{n}$ is a random perturbation with prescribed variance. If the variance is chosen to be very large, then the random walk sampler becomes similar to the independent sampler. Although the random walk sampler allows us to accept more realizations, it often gets stuck in the neighborhood of a local maximum of the target distribution. For both proposal distributions, we have observed consistently several times of increase in the acceptance rate when the preconditioned MCMC is used.

We present numerical experiments for two-phase flow simulations. We consider $\mu_{o} / \mu_{w}=5, k_{r w}(S)=S^{2}$, $k_{r o}(S)=(1-S)^{2}, 40 \times 40$ fine-scale log-normal permeability field with $L_{x}=L_{y}=0.1$ and $10 \times 10$ coarse-scale models. In Figure 10, the acceptance rate for $\sigma_{f}^{2}=0.001$ is plotted for various values of $\sigma_{c}$. We observe several times increase in the acceptance rate. The preconditioned MCMC method accepts the same number of samples as in the full MCMC with only $10 \%$ of the fine-scale runs. To study the relative convergence of the preconditioned MCMC method, in Figure 11 we plot fractional flow error for both full and preconditioned MCMC simulations. It can be seen from this figure that both the full and preconditioned MCMC methods reach the steady state within 20 accepted iterations. This indicates that both direct and preconditioned MCMC methods have similar convergence properties.

An important type of proposal distribution can be derived from the Langevin diffusion, as proposed by Grenander and Miller [21]. The Langevin diffusion is defined by the stochastic differential equation

$$
d k(\tau)=\frac{1}{2} \nabla \log \pi(k(\tau)) d \tau+d W_{\tau},
$$

where $W_{\tau}$ is the standard Brownian motion vector with independent components. It can be shown that the diffusion process $k(\tau)$ has $\pi(k)$ as its stationary distribution. The actual implementation of the Langevin 


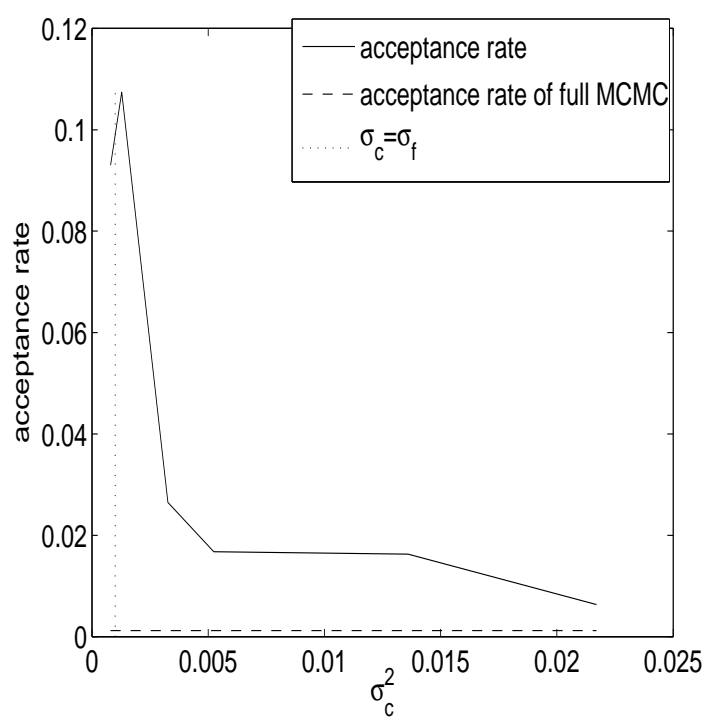

Figure 10: Acceptance rate vs. coarse-scale precision of MCMC using $10 \times 10$ coarse-scale models $\left(\sigma_{f}^{2}=\right.$ $0.001)$.

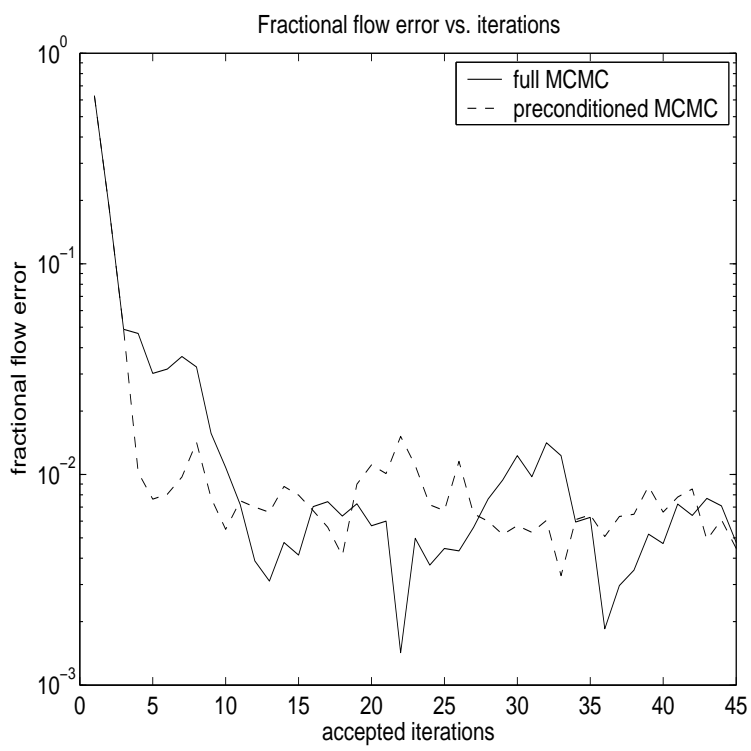

Figure 11: Fractional flow error vs. accepted iterations. 
diffusion requires a discretization of the equation (4.16),

$$
k_{n+1}=k_{n}+\frac{\Delta \tau}{2} \nabla \log \pi\left(k_{n}\right)+\sqrt{\Delta \tau} \epsilon_{n}
$$

where $\epsilon_{n}$ are independent standard normal distributions. However, the discrete solution $k_{n}$ can have vastly different asymptotic behavior from the continuous diffusion process $k(t)$ [29]. In general, the discrete solution $k_{n}$ does not necessarily have $\pi(k)$ as its stationary distribution. Instead of taking $k_{n}$ as samples directly, we use them as proposals which are further tested and corrected by the Metropolis accept and reject step. In particular, we choose the instrumental proposal $q\left(Y \mid k_{n}\right)$ as

$$
Y=k_{n}+\frac{\Delta \tau}{2} \nabla \log \pi\left(k_{n}\right)+\sqrt{\Delta \tau} \epsilon_{n}
$$

The transition distribution of the proposal (4.17) is

$$
\begin{aligned}
& q\left(Y \mid k_{n}\right) \propto \exp \left(-\frac{\left\|Y-k_{n}-\frac{\Delta \tau}{2} \nabla \log \pi\left(k_{n}\right)\right\|^{2}}{2 \Delta \tau}\right) \\
& q\left(k_{n} \mid Y\right) \propto \exp \left(-\frac{\left\|k_{n}-Y-\frac{\Delta \tau}{2} \nabla \log \pi(Y)\right\|^{2}}{2 \Delta \tau}\right) .
\end{aligned}
$$

The use of the gradient information in inverse problems for subsurface characterization is not new. In [28], the authors use the gradient information of the target distribution and propose the randomized maximum likelihood method. This approach uses unconditional realizations of the production and permeability data and solves a deterministic gradient-based minimization problem. The solution of this minimization problem is taken as a proposal and is accepted with probability one because the rigorous acceptance probability is very difficult to estimate. Besides the need to solve a gradient-based inverse problem, this method does not properly sample the posterior distribution. Thus, developing efficient rigorous MCMC calculations with high acceptance rate remains a challenging problem. Though the Langevin proposal (4.17) resembles the proposal used in the randomized maximum likelihood method, it is more efficient and rigorous, e.g., one can compute the acceptance probability. The Langevin algorithms also allow us to achieve high acceptance rates. However, the gradient computations are very expensive. In [10], we, jointly with P. Dostert and W. Luo, propose to use the coarse-scale solutions in the calculations of the gradients to speed up the Langevin algorithms. Next, we briefly describe this algorithm.

\section{Algorithm II (Preconditioned coarse-gradient Langevin algorithm)}

- Step 1. At $k_{n}$, generate a trial proposal $Y$ from the coarse Langevin distribution $q^{*}\left(Y \mid k_{n}\right)$.

- Step 2. Take the proposal $k$ as

$$
k=\left\{\begin{array}{l}
Y \text { with probability } g\left(k_{n}, Y\right), \\
k_{n} \text { with probability } 1-g\left(k_{n}, Y\right),
\end{array}\right.
$$

where

$$
g\left(k_{n}, Y\right)=\min \left(1, \frac{q^{*}\left(k_{n} \mid Y\right) \pi^{*}(Y)}{q^{*}\left(Y \mid k_{n}\right) \pi^{*}\left(k_{n}\right)}\right) .
$$

Here $\pi^{*}$ stands for a coarse-scale approximation of $\pi$. Therefore, the proposal $k$ is generated from the effective instrumental distribution

$$
Q\left(k \mid k_{n}\right)=g\left(k_{n}, k\right) q^{*}\left(k \mid k_{n}\right)+\left(1-\int g\left(k_{n}, k\right) q^{*}\left(k \mid k_{n}\right) d k\right) \delta_{k_{n}}(k) .
$$


- Step 3. Accept $k$ as a sample with probability

$$
\rho\left(k_{n}, k\right)=\min \left(1, \frac{Q\left(k_{n} \mid k\right) \pi(k)}{Q\left(k \mid k_{n}\right) \pi\left(k_{n}\right)}\right),
$$

i.e., $k_{n+1}=k$ with probability $\rho\left(k_{n}, k\right)$, and $k_{n+1}=k_{n}$ with probability $1-\rho\left(k_{n}, k\right)$.

The transition distribution of the coarse-grid proposal is

$$
\begin{aligned}
& q^{*}\left(Y \mid k_{n}\right) \propto \exp \left(-\frac{\left\|Y-k_{n}-\frac{\Delta \tau}{2} \nabla \log \pi^{*}\left(k_{n}\right)\right\|^{2}}{2 \Delta \tau}\right), \\
& q^{*}\left(k_{n} \mid Y\right) \propto \exp \left(-\frac{\left\|k_{n}-Y-\frac{\Delta \tau}{2} \nabla \log \pi^{*}(Y)\right\|^{2}}{2 \Delta \tau}\right) .
\end{aligned}
$$

To compute the gradient of $\pi^{*}\left(k_{n}\right)$ numerically, we only need to solve the PDE system (2.1)-(2.3) on the coarse-grid. Again, we use an inexpensive approximate model that combines MsFVEM for the pressure equation and (3.3) for the saturation equation. The coarse-scale distribution $\pi^{*}(k)$ serves as a regularization of the original fine-scale distribution $\pi(k)$. By replacing the fine-scale gradient with the coarse-scale gradient, we can reduce the computational cost dramatically but still direct the proposals to regions with larger probabilities. The analysis of preconditioned coarse-gradient Langevin algorithm is presented in [10].

Next we present some numerical results. The permeability field is assumed to have Gaussian covariance with $L_{x}=L_{y}=0.3, \sigma^{2}=2$. We consider $\mu_{o} / \mu_{w}=5, k_{r w}(S)=S^{2}, k_{r o}(S)=(1-S)^{2}$. Nine values of the permeability field are assumed to be 1 . We assume side-to-side boundary conditions. In Karhunen-Loeve expansion, first 20 eigenfunctions are kept. In our computations the fine grid is $60 \times 60$, and the coarse-grid is $6 \times 6, \sigma_{f}^{2}=0.003$ and $\Delta \tau=0.05$. In Figure 12, the response surface for $\pi$ and $\pi^{*}$ (defined by (4.3) and (4.3)) restricted to two dimensional hyperplane in $\theta$ are shown. We observe that $\pi^{*}$ approximates $\pi$ very well in large scales, though $\pi$ has more variations on small scales. The proximity of $\pi$ and $\pi^{*}$ guarantees the efficient sampling if $\pi^{*}$ is used in preconditioning step. In Figure 13, the acceptance rate for the direct fine-scale Langevin and the preconditioned coarse-gradient Langevin are compared. As we see from this figure, the acceptance rates of the preconditioned coarse-gradient Langevin is slightly higher than that of the direct Langevin algorithm. This indicates that preconditioned coarse-gradient Langevin algorithm accepts approximately the same number of proposals as the direct Langevin algorithm. However, the gradient computations in the preconditioned coarse-gradient Langevin algorithm is performed using the solution on the coarse grid. Thus, the speedup of the preconditioned coarse-gradient Langevin algorithm approximately equals to the speedup gained in coarse-scale computations. If the fine-scale model is upscaled 6 times in each direction, as we did in the numerical experiment, then the coarse-scale model is at least 36 times faster than the fine-scale model. Indeed, at each time step solving the pressure equation on the coarse grid is approximately 36 times faster than on the fine grid. Moreover, the update of the saturation is performed with the time step corresponding to the coarse mesh size. Thus, we perform less pressure updates in the coarse-scale simulations compared to the fine-scale simulations. As a result, we have observed two order of magnitude of CPU speedup if the preconditioned coarse-gradient Langevin algorithm is used. In Figure 14, the fractional flow errors and fractional flows are plotted. The results in Figure 14 indicates that Langevin and direct MCMC methods have similar convergence properties. In the computations, $\Delta \tau=0.05$ is kept fixed. Because the fine-scale response surface, $\pi$, varies on smaller scales, we observe some irregular behavior in fractional flow error for early proposals. On the right figure, the fractional flow curves are plotted for accepted realizations after the chain has reached a steady state. We observe that these samples provide nearly the same fractional flow response as the reference permeability field. More discussion and numerical examples on the performance of coarse-gradient Langevin algorithms can be found in [10].

The MCMC method used in the paper employs multiscale finite volume element methods in the preconditioning step. If a proposal is accepted by the preconditioning step, the proposed algorithms compute the fine-scale solutions corresponding to the proposed permeability field. At this stage, we have already precomputed basis functions that can be further used to re-construct the velocity field on the fine-scale. 

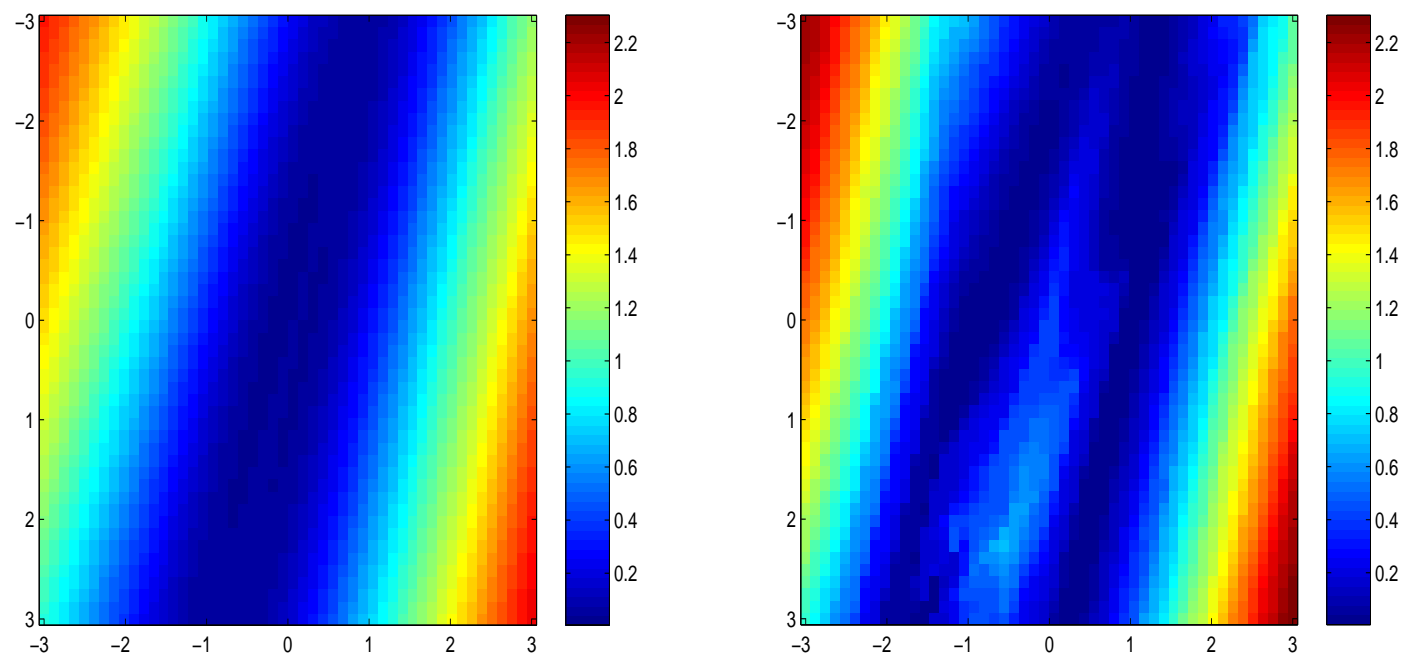

Figure 12: Left: Coarse-scale response surface $\pi^{*}$ restricted to 2-D hyperplane. Right: Fine-scale response surface $\pi$ restricted to the same 2-D hyperplane

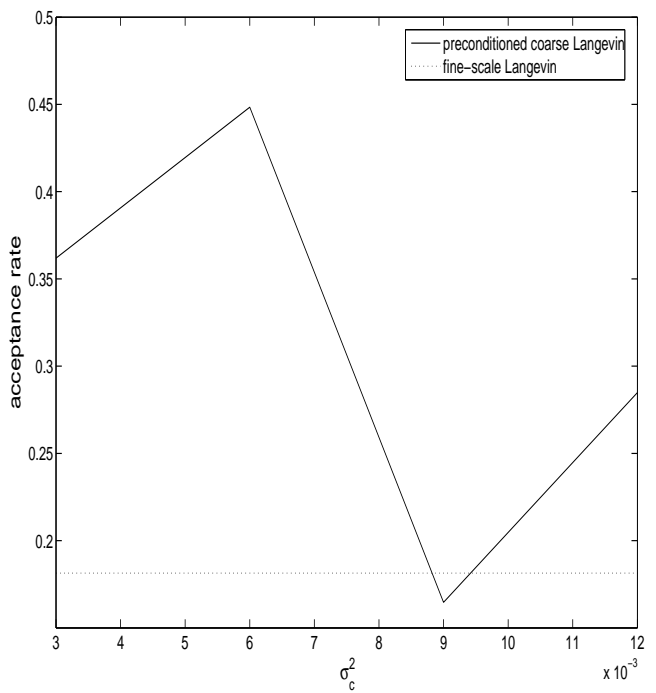

Figure 13: Acceptance rate comparison for direct fine-scale Langevin and preconditioned coarse-gradient Langevin algorithms for two-phase flow, $\Delta \tau=0.05, \sigma_{f}^{2}=0.003$. 

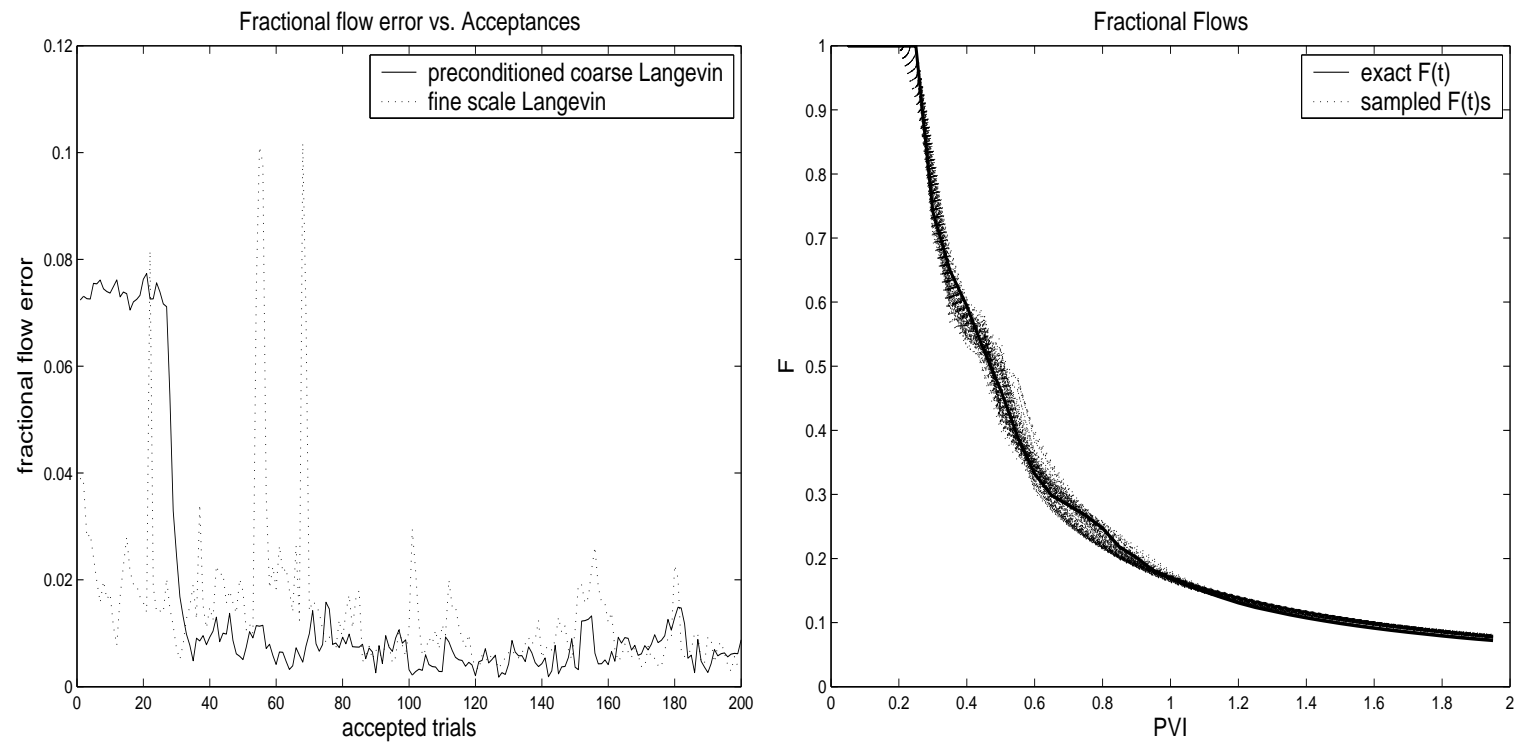

Figure 14: Left: The fractional flow errors for direct fine-scale Langevin algorithm compared with preconditioned coarse-gradient Langevin algorithm. Right: The fractional flow curves of sampled realizations and the reference fractional flow. In these numerical tests, $\Delta \tau=0.05, \sigma_{f}^{2}=0.003$ and $6 \times 6$ coarse-scale model is used.

Then the transport equation can be solved on the fine-grid coupled with the coarse-grid pressure equation. This approach provides an accurate approximation to the production data on the fine-grid (cf. $[18,25,1])$ and can be used to replace the fine-scale computation in the last stage. In this procedure, the basis functions are not updated in time, or updated only in a few coarse blocks. Thus the fine-scale computation in the last stage of MCMC algorithms can also be implemented fast. Since the basis functions from the first-stage is re-used for the fine-scale computation, this combined multiscale approach can be very efficient for our sampling problem.

\section{Conclusion}

In this paper, we discuss some applications of multiscale finite element methods to two-phase immiscible flow simulations in heterogeneous porous media. We discuss some extensions of multiscale finite element methods which take into account some limited global information. These methods are well suited for channelized porous media, where the long-range effects are important. This is typical for some recent benchmark tests, such as the SPE comparative solution project [8], where porous media has a channelized structure. The applications of multiscale finite element methods to inverse problems arisen in subsurface characterization is also discussed in the paper. In particular, we show that using inexpensive coarse-scale models involving multiscale finite element methods, one can perform efficient sampling from a complicated posterior distribution arisen in inverse problems.

The MCMC method used in the paper employs multiscale finite volume element methods in the preconditioning step. If a proposal is accepted by the preconditioning step, the proposed algorithms compute the fine-scale solutions corresponding to the proposed permeability field. At this stage, we have already precomputed basis functions that can be further used to re-construct the velocity field on the fine-scale. Then the transport equation can be solved on the fine-grid coupled with the coarse-grid pressure equation. This approach provides an accurate approximation to the production data on the fine-grid and can be used to replace the fine-scale computation in the preconditioning stage. In this procedure, the basis functions are not updated in time, or updated only in a few coarse blocks. Thus the fine-scale computation in the 
last stage of MCMC algorithms can also be implemented fast. Since the basis functions from the first-stage is re-used for the fine-scale computation, this combined multiscale approach can be very efficient for our sampling problem.

\section{Acknowledgments}

We would like to thank Paul Dostert, Victor Ginting and Wuan Luo for their help in preparing this manuscript. The research of the first author is partially supported by NSF grants DMS-0327713 and DOE grant DE-FG02-05ER25669. The research of the second author is partially supported by NSF ITR Grant No. ACI-0204932 and NSF FRG Grant No. DMS-0353838.

\section{References}

[1] J. AARNES, On the use of a mixed multiscale finite element method for greater flexibility and increased speed or improved accuracy in reservoir simulation, SIAM MMS, 2 (2004), pp. 421-439.

[2] T. Arbogast, Implementation of a locally conservative numerical subgrid upscaling scheme for twophase Darcy flow, Comput. Geosci., 6 (2002), pp. 453-481.

[3] I. Babuška, G. Caloz, and E. Osborn, Special finite element methods for a class of second order elliptic problems with rough coefficients, SIAM J. Numer. Anal., 31 (1994), pp. 945-981.

[4] I. BABUšKKA AND E. OsBorn, Generalized finite element methods: Their performance and their relation to mixed methods, SIAM J. Numer. Anal., 20 (1983), pp. 510-536.

[5] F. BREzzI, Interacting with the subgrid world, in Numerical analysis 1999 (Dundee), Chapman \& Hall/CRC, Boca Raton, FL, 2000, pp. 69-82.

[6] Y. Chen, L. J. Durlofsky, M. Gerritsen, and X. H. Wen, A coupled local-global upscaling approach for simulating flow in highly heterogeneous formations, Advances in Water Resources, 26 (2003), pp. 1041-1060.

[7] Z. Chen And T. Y. Hou, A mixed multiscale finite element method for elliptic problems with oscillating coefficients, Math. Comp., 72 (2003), pp. 541-576 (electronic).

[8] M. Christie And M. Blunt, Tenth spe comparative solution project: A comparison of upscaling techniques, SPE Reser. Eval. Eng., 4 (2001), pp. 308-317.

[9] C. V. Deutsch And A. G. Journel, GSLIB: Geostatistical software library and user's guide, 2nd edition, Oxford University Press, New York, 1998.

[10] P. Dostert, Y. Efendiev, T. Hou, And W. Luo, Coarse-gradient Langevin algorithms for dynamic data integration and uncertainty quantification, J. Computational Physics, Submitted

[11] L. J. Durlofsky, Coarse scale models of two phase flow in heterogeneous reservoirs: Volume averaged equations and their relationship to the existing upscaling techniques, Computational Geosciences, 2 (1998), pp. 73-92.

[12] L. J. Durlofsky, R. A. Behrens, R. C. Jones, And A. Bernath, Scale up of heterogeneous three dimensional reservoir descriptions, SPE paper 30709, (1996).

[13] L. J. Durlofsky, R. C. Jones, and W. J. Milliken, A nonuniform coarsening approach for the scale up of displacement processes in heterogeneous media, Advances in Water Resources, 20 (1997), pp. 335-347. 
[14] W. E And E. Engquist, The heterogeneous multi-scale methods, Comm. Math. Sci., 1(1) (2003).

[15] Y. Efendiev and L. Durlofsky. Generalized convection-diffusion model for subgrid transport in porous media. SIAM Multiscale Modeling and Simulation, 1(3):504-526, 2003.

[16] Y. R. Efendiev, L. J. Durlofsky, And S. H. Lee, Modeling of subgrid effects in coarse scale simulations of transport in heterogeneous porous media, Water Resour. Res., 36 (2000), pp. 2031-2041.

[17] Y. Efendiev, T. Hou, And V. Ginting, Multiscale finite element methods for nonlinear problems and their applications, Comm. Math. Sci., 2 (2004).

[18] Y. Efendiev, V. Ginting, T. Hou, And R. Ewing, Accurate multiscale finite element methods for two-phase flow simulations. Submitted.

[19] Y. Efendiev, T. Hou And W. Luo, Preconditioning Markov chain Monte Carlo simulations using coarse-scale models. SIAM Scientic Computation. Submitted.

[20] J. Glimm And D. H. Sharp, Prediction and the quantification of uncertainty, Phys. D, 133 (1999), pp. 152-170. Predictability: quantifying uncertainty in models of complex phenomena (Los Alamos, NM, 1998).

[21] U. Grenander And M.I. Miller, Representations of knowledge in complex systems (with discussion), J. R. Statist. Soc. B, 56 (1994), 549-603.

[22] T. Y. Hou And X. H. Wu, A multiscale finite element method for elliptic problems in composite materials and porous media, Journal of Computational Physics, 134 (1997), pp. 169-189.

[23] T. Y. Hou AND X. H. Wu, A multiscale finite element method for PDEs with oscillatory coefficients, Notes on Numerical Fluid Mechanics, Vol. 70, pp 58-69, 1999, Vieweg-Verlag.

[24] T. Hughes, G. Feijoo, L. Mazzei, And J. Quincy, The variational multiscale method - a paradigm for computational mechanics, Comput. Methods Appl. Mech. Engrg, 166 (1998), pp. 3-24.

[25] P. Jenny, S. Lee, And H. TChelepi,Adaptive multi-scale finite volume method for multi-phase flow and transport in porous media, Multiscale Modeling and Simulation, 3 (2005), pp. 30-64.

[26] A.-M. Matache And C. Schwab, Homogenization via p-FEM for problems with microstructure, in Proceedings of the Fourth International Conference on Spectral and High Order Methods (ICOSAHOM 1998) (Herzliya), vol. 33, 2000, pp. 43-59.

[27] D. Oliver, L. Cunha, And A. Reynolds, Markov chain Monte Carlo methods for conditioning a permeability field to pressure data, Mathematical Geology, 29 (1997).

[28] D. Oliver, N. He, And A. Reynolds, Conditioning permeability fields to pressure data. 5th European conference on the mathematics of oil recovery, Leoben, Austria, 3-6 September, 1996.

[29] C. Robert and G. Casella, Monte Carlo Statistical Methods, Springer-Verlag, New-York, 1999.

[30] T. Strinopoulos, Upscaling of immiscible two-phase flows in an adaptive frame, $\mathrm{PhD}$ thesis, California Institute of Technology, Pasadena, 2005.

[31] A. Westhead, Upscaling for Two-phase Flows in Porous Media. , PhD thesis, California Institute of Technology, Pasadena, 2005.

[32] E. Wong, Stochastic Processes in Information and Dynamical Systems, MCGraw-Hill, 1971. 